Айда-заде К.Р. ${ }^{1}$, Рзаева С.Г. ${ }^{2}$, Талыбов С.Г. ${ }^{3}$

${ }_{1,2,3}$ Институт Систем Управления НАНА, Баку, Азербайджан

${ }^{3}$ Бакинский Государственный Университет, Баку, Азербайджан

1'kamil_aydazade@rambler.ru, ${ }^{2}$ sonarza@yahoo.com, ${ }^{3}$ saxavat@yahoo.com

\title{
АВТОМАТИЗИРОВАННАЯ ДИАЛОГОВАЯ СИСТЕМА ДЛЯ СОСТАВЛЕНИЯ РАСПИСАНИЯ ЗАНЯТИЙ В ВЫСШИХ УЧЕБНЫХ ЗАВЕДЕНИЯХ
}

Поступила: 08.07.2019

Исправлена: 16.09.2019

Принята: 15.10.2019

В статье описывается разработанная автоматизированная диалоговая система проиесса составления и анализа качества расписания занятий в высших учебных заведениях. Приведены технологическая и математическая постановки задачи составления расписания занятий, являющегося важным элементом организации и проведения учебного проиесса для всех учебных заведений. Описаны структура, способы хранения и обработки входных данных. Предложено математическое описание требований и критериев оченки расписания. В качестве интерфейса программы с пользователями используется концепция «светофора». Описывается процесс расстановки занятий по ячейкам таблицы расписания занятий в соответствии с иветом выбранной ячейки. Показаны достоинства системы, обеспечивающие облегчение и ускорение работы пользователя, исключающие ошибки при составлении расписания. Результатом работы системы является расписание, удовлетворяющее всем основным требованиям, предъявляемым самим учебным прочессом и по возможности удовлетворяюшим нестрогим требованиям - пожеланиям педагогов, студентов, несущественно влияющим на учебный процесс.

Ключевые слова: расписание занятий, учебный процесс, информационная система, требования к системе, «светофор», ячейка таблицы, интерфейс пользователя.

\section{Введение}

Важной задачей при организации и проведении учебного процесса является этап составления расписания занятий. Оно составляется на определенный период времени обучения. Как правило, в вузах расписание в основном составляется на один семестр, хотя может быть необходимость составления расписания и на другие периоды.

Расписание занятий регламентирует работу преподавателей, студентов, обеспечивает выполнение учебного плана и рабочих программ. От качественно составленного расписания существенно зависит и качество учебного процесса в вузе.

Разработка расписания представляет собой сложный и трудоемкий процесс, так как задача составления расписания характеризуется большим объемом данных и множеством требований (ограничений), предъявляемых к расписанию. Это и загруженность студентов и преподавателей, и ограниченность, а как правило, нехватка аудиторного фонда и его специализация, а также необходимость учета методических, организационных, педагогических и медицинских требований и условий, накладываемых на готовое расписание. Кроме того, следует учесть пожелания преподавателей и, в первую очередь, преподавателейсовместителей. Причем зачастую требования и пожелания участников этого процесса (преподавателей и студентов) антагонистичны. Все это ставит под сомнение возможность составления расписания, удовлетворяющего всем требованиям и пожеланиям. С другой стороны, очень усложняются процесс составления расписания и контроль его качества [1-3].

С математической точки зрения задача составления расписания представляет собой сложную комбинаторную задачу с большим числом переменных и ограничений, относящуюся к NP-полным проблемам дискретной математики. При ее решении не обойтись без автоматизации рутинных процессов для подготовки входной информации, 
осуществляющейся при ручном составлении расписания [4-5].

Одно из основных назначений автоматизированных систем составления расписания занятий заключается в необходимости освобождения пользователя (для краткости диспетчера учебного заведения) от рутинной работы - оценки качества расписания и проверки его на непротиворечивость и целостность. Пока еще не известен алгоритм оптимизации расписаний с учетом абсолютно всех требований для реальных случаев. Поэтому составление расписания - это поиск того решения, которое, возможно, и не является абсолютно наилучшим, но принимается всеми его участниками.

В работе приводится описание разработанной нами версии компьютерной системы составления расписания занятий в вузе в автоматизированном режиме.

\section{Основные функции системы и их описание}

Система предназначена для составления допустимого и эффективного расписания занятий в высшем учебном заведении в автоматизированном режиме при рациональном использовании аудиторного фонда.

Основными функциями разработанной системы являются: ввод и корректировка нормативно-справочной и оперативной информации, размещение занятий в аудиториях по группам и потокам и их корректировка. Важными функциями системы также являются: анализ имеющегося расписания, выявление ошибок, недостатков, противоречий и т.П., выдача рекомендаций (вариантов) корректировок.

В основе предлагаемой системы анализа и составления расписания в вузе лежит принцип «светофора», суть которого состоит в том, что вначале проводится анализ, а потом уже сам процесс составления или корректировки расписания. Поэтому при составлении или корректировке расписания диспетчером с применением данной системы ошибки исключаются - система анализирует и проверяет уже имеющееся расписание. Для работы с системой диспетчеру предлагается простой и удобный интерфейс.

\section{Постановка задачи}

Определим перечень основных требований и ограничений, предъявляемых к расписанию [6-8].

Ограничения подразделяются на жесткие и мягкие. Жесткие ограничения связаны с требованиями полного выполнения всей учебной нагрузки как преподавателями, так и учебными группами, отсутствия накладок у групп, преподавателей и аудиторий. Нарушение жестких требований приводит к нарушению учебного процесса в целом относительно группы, кафедры или факультета. Если нарушены жесткие требования, значит нужно считать, что расписание не создано.

Мягкие ограничения (пожелания преподавателей, организационные, медицинские и педагогические требования и др.) не приводят к нарушению учебного процесса, но причиняют неудобства студентам, преподавателям или руководству учебного заведения.

Постановка задачи составления расписания должна включать в себя ограничения, определяемые приведенными выше требованиями, и критерии качества. Качество расписания можно характеризовать формируемыми приведенными выше требованиями. Оценка принимаемого решения задачи по составлению расписания, или качество расписания, является, очевидно, векторной величиной. Соответственно, задача составления расписания является задачей векторной конечномерной дискретной оптимизации [9-11]. В качестве ограничений примем главные требования, а за основу критериев, которые должны быть удовлетворены в максимальной степени, могут быть взяты следующие показатели и характеристики расписания:

1. Отсутствие повторяемости предметов в течение учебного дня.

2. Допустимое количество лекций в день. 
3. Чередование предметов различных блоков.

4. Минимизация «окон» у студентов.

5. Минимизация «окон» у преподавателей.

6. Привязка аудиторий к факультетам и кафедрам.

7. Проведение занятий в специализированных аудиториях.

8. Соответствие вместимости аудиторий количественному составу студенческих групп и потоков.

9. Учет пожеланий преподавателей.

10. Минимизация переходов между зданиями.

Некоторые из этих критериев в задаче могут учитываться в качестве ограничений.

Математическая формулировка задачи с учетом всех ограничений и критериев имеет достаточно громоздкий вид. Как правило, она приводится к многоиндексной многокритериальной линейной задаче целочисленного программирования [12-16]. Из-за ее сложности она относится к NP-полным задачам целочисленного программирования. Получение ее точного решения из-за большой размерности, которую имеют реальные задачи составления расписания, практически невозможно. Поэтому нами используются эвристические методы, основанные, как правило, на использовании экспертной информации, опыта диспетчера, диалогового режима человек-компьютер, заложенные в описываемую ниже разработанную систему составления расписания.

\section{Обозначения и переменные}

Введем обозначения и определим переменные, необходимые для формирования математической постановки задачи составления расписания.

Пусть:

$\left\{f_{i}: i=1, \ldots, F\right\}$ - множество кодов факультетов вуза, число которых равно $F$.

$\left\{n_{i}: i=1, \ldots, N\right\}-$ множество кодов кафедр вуза, число которых равно $N$.

$\left\{p_{i}: i=1, \ldots, P\right\}$ - множество кодов преподавателей вуза, число которых равно $P$.

$\left\{g_{i}: i=1, \ldots, G\right\}$ - множество кодов учебных групп, число которых равно $G$.

$\left\{C^{g_{i}}\right\}$ - количество студентов в группе $g_{i}$.

$\left\{l_{i}: i=1, \ldots, L\right\}$ - множество кодов лекционных предметов, число которых равно $L$.

$\left\{z_{i}: i=1, \ldots, Z\right\}$ - множество кодов предметов практических занятий, число которых равно $Z$.

Каждый изучаемый в вузе предмет имеет свой профиль, поэтому все предметы разбиты по разным блокам, которые зададим следующим образом:

$\left\{b_{i}: i=1, \ldots, B\right\}$ - множество кодов блоков предметов в количестве $B$.

Занятия проводятся в дни, определяемые режимом работы вуза $\{d=1, \ldots, D\}-$ коды дней недели, количество которых $D$.

Каждый учебный день состоит из занятий, которые проводятся парами:

$\{t=1, \ldots, T\}-$ множество кодов учебных пар в количестве $T$.

$\left\{r_{i}: i=1, \ldots, R\right\}$ - множество кодов курсов обучения, число которых равно $R$.

Каждый курс может начинать и заканчивать занятия только с определенных временных

пар. Пусть $Q_{1 r_{i}}$ и $Q_{2 r_{i}}$ есть соответственно начало и окончание занятий для каждого курса $r_{i}$.

$\left\{k_{i}: i=1, \ldots, K\right\}$ - множество кодов учебных корпусов, число которых равно $K$. 
$\left\{a_{i k}: i=1, \ldots, A_{k}, k=1, \ldots, K\right\}$ - множество кодов аудиторий учебного корпуса $k$, число которых равно $A_{k}$.

Проведение занятий по ряду предметов возможно лишь в специализированных аудиториях:

$\left\{s_{i}: i=1, \ldots, S\right\}$ - множество кодов типов аудиторий, число которых равно $S$.

$V^{\alpha_{i k}}-$ вместимость аудитории $a_{i}$ в учебном корпусе $k$.

$S^{\alpha_{i k}}-$ код типа специализации аудитории $a_{i}$ в учебном корпусе $k$.

$f^{\alpha_{i k}}-$ код факультета, за которым закреплена аудитория с кодом $a_{i k}$.

$n^{\alpha_{i k}}-$ код кафедры, за которой закреплена аудитория с кодом $a_{i k}$.

На основании учебного плана и нагрузки преподавателей для каждой группы $g_{i}$ составляется список запланированных на неделю предметов $-U^{g_{i}}$, а для каждого преподавателя $p_{i}$ вычисляется его недельная нагрузка $-W^{p_{i}}$.

Введем следующие переменные.

Для каждого преподавателя с кодом $p_{i}$ из множества всех преподавателей введем в рассмотрение булеву переменную $x_{d t}^{p_{i}}$, где $p_{i}=1, \ldots, P, d=1, \ldots, D, t=1, \ldots, T$ :

$$
x_{d t}^{g_{i}}= \begin{cases}1, & \text { если преподаватель с кодом } p_{i} \text { в день } d \text { проводит занятие } \\ 0, & \text { ва временной паре } t,\end{cases}
$$

Для каждой группы с кодом $g_{i}$ из множества всех групп введем в рассмотрение булеву переменную $x_{d t}^{g_{i}}$, где $g_{i}=1, \ldots, G, d=1, \ldots, D, t=1, \ldots, T$ :

$$
x_{d t}^{g_{i}}= \begin{cases}1, & \text { если группа с кодом } g_{i} \text { в день } d \text { находится на занятиях } \\ 0, & \text { в пр временной паре } t,\end{cases}
$$

Для каждой аудитории с кодом $a_{i k}$ из множества всех аудиторий введем в рассмотрение булеву переменную $o_{1 d t}^{a_{k}}$, где $k=1, \ldots, K, a_{i k}=1, \ldots, A_{k}, d=1, \ldots, D, t=1, \ldots, T$ :

$$
o_{1 d t}^{a_{i k}}= \begin{cases}1, & \text { если аудитория } a_{i k} \text { корпуса } k \text { занята в день } d \\ 0, & \text { ва вр пременной паре } t\end{cases}
$$

Для каждого преподавателя с кодом $p_{i}$ из множества всех преподавателей введем в рассмотрение булеву переменную $o_{2 d t}^{p_{i}}$, где $p_{i}=1, \ldots, P, d=1, \ldots, D, t=1, \ldots, T$ :

$$
c_{o_{2}}^{p_{i}}= \begin{cases}1, & \text { если преподаватель в кодом } p_{i} \text { не желает проводить занятие } \\ 0, & \text { в день } d \text { на временновй паре } t,\end{cases}
$$

Для каждой лекции с кодом $l_{j}$ из множества всех лекций введем в рассмотрение булеву переменную $o_{3 d t l_{j}}^{g_{i}}$, где $g_{i}=1, \ldots, G, d=1, \ldots, D, t=1, \ldots, T, l_{j}=1, \ldots, L$ : 


$$
o_{3 d t_{j}}^{g_{i_{i}}}= \begin{cases}1, & \text { если группас кодом } \mathrm{g}_{\mathrm{i}} \text { в день } d \text { на временной паре } t \text { проходит } \\ 0, & \text { в прционное занятие с кодом } l_{j},\end{cases}
$$

Для каждого практического занятия с кодом $z_{j}$ из множества всех практических занятий введем в рассмотрение булеву переменную $o_{4 d t z_{j}}^{g_{g_{i}}}$, где $g_{i}=1, \ldots, G, d=1, \ldots, D$, $t=1, \ldots, T, z_{j}=1, \ldots, Z$ :

$$
o_{4 d t z_{j}}^{g_{i}}= \begin{cases}1, & \text { если группас кодом } g_{i} \text { в день } d \text { на временной паре } t \\ 0, & \text { в проходит практическое занятие с кодом } z_{j},\end{cases}
$$

Для каждой группы с кодом $g_{i}$ из множества всех групп введем в рассмотрение булеву переменную $o_{5}^{g_{i}}{ }_{d b_{j}}$, где $g_{i}=1, \ldots, G, b_{j}=1, \ldots, B, d=1, \ldots, D, t=1, \ldots, T$ :

$$
o_{5}^{g_{i}}= \begin{cases}1, & \text { если группас кодом } g_{i} \text { в день } d \text { на временной паре } t \\ 0, & \text { взучает предмет из блока с кодом } b_{j},\end{cases}
$$

Для каждой группы с кодом $p_{i}$ из множества всех групп введем в рассмотрение булеву переменную $o_{6 d t}^{g_{i}}$, где $g_{i}=1, \ldots, G, d=1, \ldots, D, t=1, \ldots, T$ :

$$
o_{6 d t}^{g_{i}}= \begin{cases}1, & \text { если группас кодом } g_{i} \text { в день } d \text { на временной паре } t \text { проходит } \\ 0, & \text { в прционное занятие },\end{cases}
$$

Введем нижеследующие переменные:

$y_{1 d t}^{g_{i}}$ - код факультета, группа которого с кодом $g_{i}$ находится на занятиях в день $d$ на временной паре $t$.

$y_{2 d t}^{p_{i}}$-код кафедры, преподаватель которой с кодом $p_{i}$ проводит занятие в день $d$ на временной паре $t$.

$y_{3 d t}^{l_{i}}-$ код специализации аудитории, которая необходима для лекции с кодом $l_{i}$ в день $d$ на временной паре $t$.

$y_{4 d t}^{z_{i}}-$ код специализации аудитории, которая необходима для практического занятия с кодом $z_{i}$ в день $d$ на временной паре $t$.

$y_{5 d t}^{g_{i}}$ - код учебного корпуса, в котором группа с кодом $g_{i}$ находится на занятиях в день $d$ на временной паре $t$.

$y_{6 d t}^{p_{i}}-$ код учебного корпуса, в котором преподаватель с кодом $p_{i}$ проводит занятие в день $d$ на временной паре $t$. 


\section{Ограничения, обусловленные жесткими требованиями к расписанию}

1. Для каждой группы с кодом $g_{i}$ должны выполняться все виды аудиторной работы в течение недели:

$$
\sum_{d=1}^{D} \sum_{t=1}^{T}\left(2 * x_{d t}^{g_{i}}\right)=U^{g_{i}}, g_{i}=1, \ldots, G
$$

2. Каждый преподаватель с кодом $p_{i}$ в течение недели должен провести все занятия:

$$
\sum_{d=1}^{P} \sum_{t=1}^{T}\left(2 * x_{d t}^{p_{i}}\right)=W^{p_{i}}, p_{i}=1, \ldots, P
$$

3. Общая нагрузка на неделю по учебному заведению должна составлять:

$$
\sum_{p_{i}=1}^{P} \sum_{d=1}^{D} \sum_{t=1}^{T}\left(2 * x_{d t}^{p_{i}}\right)=\sum_{g_{j}=1}^{G} \sum_{d=1}^{D} \sum_{t=1}^{T}\left(2 * x_{d t}^{g_{j}}\right) .
$$

4. В каждый день $d$ на каждой временной паре $t$ преподаватель с кодом $p_{i}$ не может вести более одного занятия:

$$
\sum x_{d t}^{p_{i}} \leq 1, p_{i}=1, \ldots, P, d=1, \ldots, D, t=1, \ldots, T .
$$

5. В каждый день $d$ на каждой временной паре $t$ для каждой группы с кодом $g_{i}$ может проводиться не более одного занятия:

$$
\sum x_{d t}^{g_{i}} \leq 1, g_{i}=1, \ldots, G, d=1, \ldots, D, t=1, \ldots, T .
$$

6. В каждый день $d$ на каждой временной паре $t$ в каждой аудитории с кодом $a_{i k}$ может проводиться не более одного занятия:

$$
\sum_{k=1}^{K} \sum o_{1 d t}^{a_{i k}} \leq 1, k=1, \ldots, K, a_{i k}=1, \ldots, A_{k}, d=1, \ldots, D, t=1, \ldots, T .
$$

7. В каждый день $d$ на каждой временной паре $t$ общее число занятий не может превышать имеющийся в вузе аудиторный фонд:

$$
\sum_{i=1}^{G} x_{d t}^{g_{i}} \leq \sum_{k=1}^{K} A_{k}, d=1, \ldots, D, t=1, \ldots, T .
$$

\section{Математическое описание критериев качества расписания}

1. Величина отклонения (нарушения) критерия повторяемости предметов на лекционных занятиях в группах определяется величинами нарушения выполнения неравенств:

$$
\sum_{t=1}^{T} \sum o_{3 d t_{j}}^{g_{i}} \leq 1, d \in D, g_{i}=1, \ldots, G, l_{j}=1, \ldots, L
$$


Величина отклонения (нарушения) критерия повторяемости предметов на практических занятиях в группах определяется величинами нарушения выполнения неравенств:

$$
\sum_{t=1}^{T} \sum o_{4 d t z_{j}}^{g_{i}} \leq 1, d \in D, g_{i}=1, \ldots, G, z_{j}=1, \ldots, Z
$$

2. В каждый учебный день $d$ максимальное число лекционных занятий не может превышать число $\tau_{1}$, которое установлено педагогическими, организационными и медицинскими требованиями. Величина нарушения данного критерия определяется величинами отклонения (нарушения) условий:

$$
\sum_{t=1}^{T} o_{6 d t}{ }^{g_{i}} \leq \tau_{1}, g_{i}=1, \ldots, G, d \in D .
$$

3. В каждый учебный день $d$ максимальное число занятий, принадлежащих к одному учебному блоку, не может превышать число $\tau_{2}$, которое установлено педагогическими и медицинскими требованиями. Величина нарушения данного критерия определяется величинами отклонения (нарушения) условий:

$$
\sum_{t=1}^{T} o_{5 d b_{j}}^{g_{i}} \leq \tau_{2}, g_{i}=1, \ldots, G, b_{j}=1, \ldots, B, d \in D .
$$

4. Величина отклонения (нарушения) критерия «отсутствие окон» у студентов определяется величинами отклонения (нарушения) выполнения равенства:

$$
\sum_{g_{i}=1}^{G} \sum_{d=1}^{D} \sum_{t=2}^{T}\left|x_{d t}^{g_{i}}-x_{d(t-1)}^{g_{i}}\right|=0
$$

5. Величина отклонения (нарушения) критерия «отсутствие окон» у преподавателей определяется величинами отклонения (нарушения) выполнения равенства:

$$
\sum_{p_{i}=1}^{P} \sum_{d=1}^{D} \sum_{t=2}^{T}\left|x_{d t}^{p_{i}}-x_{d(t-1)}^{p_{i}}\right|=0 .
$$

6. Контроль использования аудиторий, закрепленных за кафедрой с кодом $n_{j}$. Для $n^{a_{k}} \neq 0$ должны выполняться условия:

$$
n^{a_{k}}=y_{2 d t}^{p_{j}}, p_{j}=1, \ldots, P, a_{i k}=1, \ldots, A_{k}, d \in D, t \in T .
$$

Контроль использования аудиторий, закрепленных за факультетом с кодом $f_{j}$. Для $f^{a^{a_{k}}} \neq 0$ должны выполняться условия:

$$
f^{a_{i k}}=y_{1 d t}^{g_{j}}, g_{j}=1, \ldots, G, a_{i k}=1, \ldots, A_{k}, a_{i k}=1, \ldots, A_{k}, d \in D, t \in T .
$$

7. Контроль за специализацией аудиторий для лекционных и практических занятий. Для $y_{3 d t}^{l_{j}} \neq 0$ должны выполняться условия:

$$
y_{3 d t}^{l_{j}}=S^{a_{i k}}, l_{j}=1, \ldots, L, a_{i k}=1, \ldots, A_{k}, d \in D, t \in T .
$$

Для $y_{4 d t}^{z_{j}} \neq 0$ должны выполняться условия: 


$$
y_{4 d t}^{z_{j}}=S^{a_{i k}}, z_{j}=1, \ldots, Z, a_{i k}=1, \ldots, A_{k}, d \in D, t \in T .
$$

8. В каждый день $d$ на каждой временной паре занятий $t$ количество студентов группы с кодом $g_{j}$ не должно превышать вместимость аудитории с кодом $a_{i k}$. Должны выполняться условия:

$$
V^{a_{i k}} \leq C^{g_{j}}, g_{j}=1, \ldots, G, a_{i k}=1, \ldots, A_{k} .
$$

9. За каждое пожелание преподавателя платится штраф, который зависит от статуса преподавателя. Пусть $\omega^{p_{i}}$ - статус преподавателя с кодом $p_{i}$. Тогда величина штрафа равна:

$$
\varphi=\sum_{p_{i}=1}^{P} \sum_{d=1}^{D} \sum_{t=1}^{T} \omega^{p_{i}} * o_{2 d t}{ }^{p_{i}} .
$$

10. Критерий «минимизации» переходов между зданиями для групп зависит от количественного состава групп и определяется следующим образом:

$$
\sum_{g_{i}=1}^{G} C^{g_{i}} \sum_{d=1}^{D} \sum_{t=2}^{T}\left|y_{5 d t}^{g_{i}}-y_{5 d(t-1)}^{g_{i}}\right| \rightarrow \min
$$

Критерий «минимизации» переходов между зданиями для преподавателей зависит от статуса преподавателя и определяется следующим образом:

$$
\sum_{p_{i}=1}^{P} \omega^{p_{i}} \sum_{d=1}^{D} \sum_{t=2}^{T}\left|y_{6 d t}^{p_{i}}-y_{6 d(t-1)}^{p_{i}}\right| \rightarrow \min .
$$

\section{Входные и выходные данные}

Входные данные для функционирования разработанной системы подразделяются на оперативную информацию и нормативно-справочную информацию.

Для нормативно-справочной информации организованы следующие таблицы: «Факультеты», «Кафедры», «Преподаватели», «Ученые степени преподавателей», «Должности преподавателей», «Предметы», «Блоки предметов», «Тип занятия», «Занятия, требующие специализированных аудиторий», «Учебные корпуса», «Аудитории», «Типы аудиторий», «Принадлежность аудиторий», «Учебные группы», «Потоки», «Виды мягких требований и их приоритеты». Работа по ведению нормативно-справочной информации реализуется по единому для всех таблиц унифицированному шаблону.

Для оперативной информации имеются следующие таблицы - «Нагрузка преподавателя» и «Пожелания преподавателей». Таблица «Нагрузка преподавателя» формируется на основе заявки на расписание, созданной преподавателем в соответствии с распределенной на него нагрузкой. Документ содержит следующую информацию: код преподавателя, номер группы или потока, код дисциплины, тип занятия, а также количество часов, предусмотренных для лекционных, практических или лабораторных занятий в неделю. Количество часов на неделю определяется в соответствии с продолжительностью семестра в неделях и общей нагрузкой на семестр по данному предмету.

Во входном документе «Пожелания преподавателей» приведена отмеченная преподавателем информация о «нежелательных часах» в определенные дни недели.

Программные модули для работы с нормативно-справочной и оперативной информацией реализуют функции, предназначенные для ведения базы данных, а также обеспечивающие ввод, удаление, хранение, редактирование, поиск, сортировку, фильтрацию и печать информации, которая содержится в таблицах.

Сформированное расписание, как правило, бывает представлено в базе данных в виде 
двух таблиц: расписание на верхнюю неделю и расписание на нижнюю неделю. Это связано с тем, что объем часов по некоторым предметам не кратен числу недель. Готовое расписание по учебным группам содержит следующую информацию: день, час, наименование предмета и форма его проведения (лекция, семинар, лабораторная работа), фамилия преподавателя, место проведения занятия (учебный корпус, аудитория). Расписание по преподавателям содержит следующую информацию: день, час, курс, номер группы, наименование предмета и форма его проведения (лекция, семинар, лабораторная работа), место проведения занятия (учебный корпус, аудитория).

\section{Описание работы системы}

При первоначальном запуске программы необходимо выполнить настройку констант. К числу обязательных констант относится график занятий - продолжительность учебной недели и начало и окончание занятий для каждого курса.

В начале работы диспетчер выбирает кафедру из предложенного ему списка. Затем на экране появляется список преподавателей выбранной кафедры. После выбора преподавателя диспетчеру предлагается нагрузка выбранного им преподавателя. В каждой строке данного документа содержится следующая информация: дисциплина, форма занятия (лекция, семинар, лабораторные занятия), группа, подгруппа, поток, нагрузка на семестр. Кроме того, на экране находится бланк расписания, который соответствует установленным настройкам. Форма бланка аналогична форме готового расписания. Выбрав конкретную строку, диспетчер получает на бланке расписания все возможные варианты расстановки выбранного занятия.

При закрашивании клеток таблицы учитываются требования, предъявляемые к расписанию, в соответствии с их приоритетами.

Красный цвет - STOP, диспетчер не может его использовать, т.к. данный ресурс недоступен по причине неудовлетворения главных требований. Если занятие в группе делится на несколько подгрупп, то анализируется расписание занятий педагогов, имеющих занятия в остальных подгруппах. Ячейки, соответствующие временной паре, когда заняты данные преподаватели, также закрашены в красный цвет, т.е. недоступны.

Если занятие назначается для потока, то анализируется расписание для всех групп, входящих в поток. Если хоть одна из групп уже занята на данной временной паре, то ячейки, соответствующие выбранной временной паре, также недоступны.

Если какие-либо занятия выбранного преподавателя уже назначены, то в ячейке таблицы расписания находится эта информация. Соответственно данная ячейка также является недоступной.

Желтый цвет - ресурс доступен, но есть причины, по которым занимать его нежелательно (причины - несоответствие некоторым критериям). Каждый из критериев имеет свой приоритет. При попытке использовать эту ячейку диспетчеру высвечивается список критериев, согласно которому ее использовать нежелательно. Но окончательное решение предоставлено диспетчеру. Использование ресурса является возможным, но это может повлиять на качество расписания.

Зеленый цвет - наилучшие возможные варианты для назначения выбранного занятия.

При закрашивании ячеек таблицы «Расписание» отражены занятия по «верхней» и «нижней» неделям. В этом случае ячейка делится диагональю на два треугольника. Причем каждый треугольник может быть закрашен разными цветом.

Закрашивание ячеек таблицы меняется для каждого выбранного диспетчером занятия в таблице «Нагрузка преподавателя».

Все документы и необходимые инструменты находятся на экране перед пользователем.

Расстановка занятий в ячейки таблицы «Расписание» производится методом «dragand-drop» («бери и тащи»). Выбранное занятие (строка в документе «Нагрузка преподавателя») захватывается «мышкой» и устанавливается в ячейку зеленого или 
желтого цвета. В ячейку красного цвета назначить занятие невозможно. Имеется возможность тем же методом по тем же правилам переставлять уже назначенные занятия между ячейками таблицы «Расписание». После установки занятия в ячейку таблицы «Расписание» в ней появляется информация с наименованием предмета, его видом (лекция, семинар, лабораторное занятие), номером группы или номерами групп в случае потока. После вставки выбранного занятия в ячейку количество заявленных в нагрузке часов уменьшается. А после распределения всех часов по определенному предмету и его виду соответствующая строка в таблице «Нагрузка преподавателя» становится недоступной.

Диспетчер может удалить информацию из таблицы «Расписание». В этом случае ячейка очищается, а соответствующее количество часов возвращается в таблицу «Нагрузка преподавателя». Если строка была неактивной, то она вновь становится активной.

На каждом этапе работы диспетчера с системой (ввод информации по расписанию, корректировка и т.п.) на экран выводится информация о качестве расписания и нарушенных мягких требованиях.

Следующий этап - назначение аудитории. Для выбора аудитории диспетчер нажимает кнопку «Выбор аудитории». Вначале выбирается учебный корпус, а затем предлагаются свободные на данный момент времени аудитории с информацией об их вместимости и принадлежности. Если выбранная аудитория не удовлетворяет некоторым критериям контроля аудиторного фонда - не соответствует количественному составу группы, не специализирована для данного типа занятий или закреплена за другой кафедрой или другим факультетом, - то принятие окончательного решения оставлено за диспетчером и он берет ответственность на себя: взять эту аудиторию или повторить поиск для выбора другой аудитории. После окончательного выбора аудитории информация с номером учебного корпуса и номера аудитории появляется в выбранной ячейке. В случае изменения или удаления аудитории они в дальнейшем будут помечены как свободные.

Процесс заполнения справочной информации может быть разделен между диспетчером, кафедрами и факультетами. Ввод оперативной информации может быть выполнен как самим диспетчером, так и кафедрами по сети, если таковая имеется в вузе.

Если система эксплуатируется не в первый раз, то возможен режим создания расписания на основе предыдущего.

Если функционирует информационная система вуза, то нормативно-справочная информация будет автоматически откорректирована на основании соответствующих приказов и постановлений (например, приказов о приеме на работу, увольнении, кадровых перемещениях, изменении учебного плана и др.) [17-18]. В противном случае изменения могут быть внесены как самим диспетчером, так и кафедрами и факультетами.

\section{Заключение}

Предлагаемая система учитывает особенности учебного процесса (лекции, практические и лабораторные занятия, деление на подгруппы и организацию поточных занятий и т.д.), позволяет оптимизировать использование аудиторного фонда, учитывает пожелания преподавателей. Система полностью освобождает диспетчера от анализа и контроля за правильностью расписания. А удобный интерфейс и современный дизайн сводят его составление к раскладыванию выбранных занятий в допустимые ячейки. Предлагаемая система может являться составной частью информационной системы вуза, что обеспечит использование единого информационного пространства [17-18].

Автоматизированная система составления и анализа расписания учебных занятий в вузе выполнена в интегрированной среде разработки Delphi с использованием СУБД MS Access. 


\section{Литература}

1. Ерунов В.П., Морковин И.И. Формирование оптимального расписания учебных занятий в вузе // Вестник Оренбургского государственного университета, 2001, №3, с.51-55. http://www.osu.ru/?doc=1037.

2. Naderi B. Modeling and Scheduling University Course Time Tabling Problems // International Journal of Research in Industrial Engineering 2016, vol.5, no.1-4, pp.1-15.

3. Гвоздев Б.А., Емельянов П.Г., Пак Е.В. Составление расписаний в учебных заведениях: требования, проблемы, подходы к решению // Новые технологии в образовании, 2007, №3, c.56-76.

4. Lindahl, Michael \& Mason, Andrew J. \& Stidsen, Thomas \& Sørensen, Matias. A strategic view of University timetabling // European Journal of Operational Research, 2018, vol.266, no.1, pp.35-45.

5. Гафаров Е.Р., Лазарев А.А. Математические методы оптимизации при составлении учебного расписания. // Новые информационные технологии в образовании: сб. науч. тр., М.: 1С-Паблишинг, 2013, ч. 2, с.51-55.

6. Хасухаджиев А.С. Формирование системы показателей для автоматизации учебного расписания типового вуза // Вестник АГТУ. Сер. Управление, вычислительная техника и информатика, 2017, №3, с.7-19.

7. Верёвкин В.И., Исмагилова О.М., Атавин Т.А. Автоматизированное составление расписания учебных занятий вуза с учетом трудности дисциплин и утомляемости студентов // Докл. Томск. гос. ун-та систем управления и радиоэлектроники, 2009, №1 (19), ч. 1, с.221-225.

8. Клеванский Н.Н., Пузанов А.А., Костин С.А. Анализ требований и ограничений в задаче составления расписаний // Образовательные технологии: Межвуз. сб. научн. тр., вып. 12, Воронеж: Центр.-Черноземн. книжн. изд-во, 2004, с.164-168.

9. Попов Г.А. Формализация задачи составления расписания в высшем учебном заведении // Вестник АГТУ, 2006, №1(30), с.120-140.

10. Applebee S., Aschinger M., Bucur A., Edmonds H., Hungerländer P. and Maier K. New Constraints and Features for the University Course Timetabling Problem / Operations Research Proceedings 2016. Selected Papers of the Annual International Conference of the German Operations Research Society (GOR), Helmut Schmidt University Hamburg, Germany, August 30-September 2, 2016, pp.95-101.

11. Снитюк В.Е., Сипко Е.Н. Штрафные функции в задаче составления расписания занятий. // Математичні машини і системи, 2015, №3, с.158-163.

12. Безгинов А.Н., Трегубов С.Ю. Обзор существующих методов составления расписания // Информационные технологии и программирование: межвузовский сборник статей, 2005, выпуск 2(14), М.: МГИУ, с.5-18.

13. Воробович Н.П., Лопатеева О.Н. Методологические основы создания автоматизированной системы формирования учебного расписания занятий в высшем учебном заведении / М-во сел. хоз-ва РФ, Краснояр. гос. аграр. ун-т., Красноярск: КрасГАУ, 2008, 175 с.

14. Кузнецов А.А. Разработка автоматизированной системы составления расписания для педагогического вуза // Современные научные исследования и инновации, 2016, №9, c.93-95. http://web.snauka.ru/issues/2016/09/67427.

15. Aizam N. A. H., Uvaraja V. Generic Model for Timetabling Problems by Integer Linear Programmimg Approach // World Academy of Science, Engineering and Technology, International Journal of Mathematical, Computational, Physical, Electrical and Computer Engineering, 2015, vol. 9, no.12, pp.668-675.

16. Клеванский Н.Н. Алгоритмы формирования расписания занятий высших учебных заведений // Ж. Фундаментальные исследования, 2017, №10 (часть 3), с.454-458. 
17. Talibov S., Rzayeva S. The Project of creation system «ELECTRONIC UNIVERSITY»/ The Third International Conference «Problems of Cybernetics and Informatics», 2010, Baku, Azerbaijan, vol.1, pp.91-93.

18. Humbatov R.T., Ayda-zade K.R., Manafli O.M., Talibov S.Q., Rzayeva S.H. Azərbaycan Dövlət Neft Akademiyası timsalında «TM» AİS-nn işlənmə layihəsi / «İnformatika, informasiya texnoloqiyalarının təhsildə tətbiqi məsələləri» respublika elmi konfransının materialları, Bak1, 22-23 May 2007, s.19-22.

\section{UOT 004.78}

Ayda-zadə Kamil R. ${ }^{1}$, Rzayeva Sona H. ${ }^{2}$, Talıbov Səxavət Q. ${ }^{3}$

${ }_{1,2,3}$ AMEA İdarəetmə Sistemləri İnstitutu, Bak1, Azərbaycan

${ }^{3}$ Bakı Dövlət Universiteti, Bakı, Azərbaycan

${ }^{1}$ kamil_aydazade@ rambler.ru, ${ }^{2}$ sonarza@yahoo.com, ${ }^{3}$ saxavat@yahoo.com

\section{Ali təhsil müəssisələrində dərs cədvəlinin tərtibi üçün avtomatlaşdırılmış dialoq sistemi}

Məqalədə ali təhsil müəssisələrində dərs cədvəllərinin tərtib edilməsi və keyfiyyətinin təhlili prosesi üçün işlənib hazırlanmış avtomatlaşdırılmış dialoq sistemi təsvir edilmişdir. Bütün ali təhsil müəssisələrində tədris prosesinin keçirilməsi və təşkilinin əsas elementi olan dərs cədvəllərinin tərtibi məsələsinin texnoloji və riyazi qoyuluşu verilmişdir. Giriş məlumatlarının strukturu, saxlanması və emalı üsulları təsvir edilmişdir. Dərs cədvəllərinə olan tələblər və qiymətləndirmə meyarlarının riyazi təsviri təklif edilmişdir. Proqram ilə istifadəçi arasında interfeys qismində "işıqfor" konsepsiyasından istifadə olunur. Seçilmiş xananın rənginə uyğun olaraq dərs cədvəlinin xanaları üzrə dərslərin bölüşdürülməsi prosesi təsvir olunur. Dərs cədvəllərinin tərtibi zamanı meydana çıxan səhvləri aradan qaldırılmağa, cədvəl tərtibi zamanı istifadəçinin işini sürətləndirməyə və asanlaşdırmağa imkan verən sistemin üstünlükləri göstərilmişdir. Sistemdən istifadə nəticəsində təhsil prosesininin özünə olan bütün əsas tələblərin və imkan daxilində tədris prosesinə çox da təsir etməyən qeyri-ciddi tələblərin (pedaqoqların, tələbələrin istəklərinin) yerinə yetirməsinə imkan verən cədvəl tərtib etmək mümkün olur.

Açar sözlor: sinif cadvali, tahsil prosesi, informasiya sistemi, sistema olan talablar, "işlqfor", cadval xanası, istifadaçi interfeysi.

\section{Kamil R. Ayda-zade ${ }^{1}$, Sona H. Rzayeva ${ }^{2}$, Sakhavat G. Talibov ${ }^{3}$}

${ }^{1,2,3}$ Institute of Control Systems of ANAS, Baku, Azerbaijan

${ }^{3}$ Baku State University, Baku, Azerbaijan

1'kamil_aydazade@rambler.ru, ${ }^{2}$ sonarza@yahoo.com, ${ }^{3}$ saxavat@yahoo.com

\section{Automated dialog system for compilation of schedule lessons in high school}

The article describes the developed automated interactive system for the process of compiling and analyzing the quality of the schedule of classes in higher educational institutions. The technological and mathematical formulations of the task of scheduling classes, which are an important element in organizing and conducting the educational process for all educational institutions, are given. The structure, methods of storage and processing of input data are described. A mathematical model of requirements and criteria is proposed. As a program interface, the concept of a «traffic light» is proposed. The process of placement of classes in the cells of the schedule table in accordance with the color of the selected cell is described in detail. The result of the system's work is a schedule that satisfies all the basic requirements of the educational process itself and, if possible, meets non-stringent requirements - the wishes of teachers, students which do not significantly affect the educational process.

Keywords: class schedule, educational process, information system, requirements to system, «traffic light», table cell, user interface. 